September 2001

\title{
Calibration and the Volatility of Labor: A Cautionary Note*
}

\begin{abstract}
A key parameter in real business cycle models is the weight on the utility of leisure. Typically this parameter is chosen so that the steady-state level of work activity matches the corresponding measure in the data, i.e. the amount of time workers spend in market activity. While the calibration of this parameter is often highlighted in business cycle research, this paper demonstrates that this parameter has no influence on equilibrium characteristics of the Hansen (1985) indivisible labor model, when solved using traditional methods. Hence, the functional form of utility rather than the parameterization of utility is the critical factor.
\end{abstract}

- JEL Classification: E32, C68

- Keywords: calibration, real business cycles

Kevin D. Salyer

Department of Economics

U. C. Davis

One Shields Ave.

Davis, CA 95616-8578

Contact Information:

phone: (530) 752-8359; e-mail: kdsalyer@ucdavis.edu

*I am indebted to Parantap Basu and Kevin Hoover for insightful comments and suggestions. 


\section{Introduction}

The empirical methodology of quantitative macroeconomics is now firmly established: construct a general equilibrium model with explicit functional forms for preferences and technology, calibrate the parameters of these functions so that features of the model (e.g. characteristics of steady-state equilibrium) match key characteristics of the data, and then use numerical approximation methods to construct the equilibrium of the model. By generating artificial data from the solution, one can study the equilibrium characteristics of the model and compare these to the data equivalents.

While this approach has improved the profession's understanding of the economy, it is also the case that, because one must use numerical methods to solve the model, the analysis often comes at the expense of intuition. With the intent of improving the intuition behind these models, this note illustrates that the standard procedure for calibrating and solving a standard real business cycle model implies that the importance of leisure in agent's utility function has no implications for the cyclical behavior of labor.

This point is established using the standard indivisible labor model due to Hansen (1985). This model, in which utility at the aggregate level is linear in leisure, was a critical development in the real business cycle approach since it improved the model's ability to match the cyclical volatility of labor. That is, standard preferences with diminishing marginal utility of leisure produced too little volatility; linear preferences produced behavior more in line with observation. This story, of course, is well known. What does not seem to be as well appreciated, however, is that the cyclical behavior of labor in this model is not affected by 
the weight on leisure in the utility function under the proviso that the model's remaining parameters are calibrated in the usual fashion. The implication is that the functional form and not the parameterization of preferences is the critical feature in determining equilibrium labor characteristics.

\section{The Model}

To show this, I start with the standard RBC model with indivisible labor written as a social planner problem:

$$
\max _{\left\{c_{t}, h_{t}, k_{t+1}\right\}_{t=0}^{\infty}} E_{0}\left\{\sum_{t=0}^{\infty} \beta^{t}\left[\ln c_{t}+A\left(1-h_{t}\right)\right]\right\}
$$

subject to:

$$
z_{t} k_{t}^{\alpha} h_{t}^{1-\alpha}+(1-\delta) k_{t}=c_{t}+k_{t+1}
$$

Current consumption is $c_{t}, h_{t}$ is labor, and $k_{t}$ is beginning of period capital stock. Output in each period is denoted $y_{t}=z_{t} k_{t}^{\alpha} h_{t}^{1-\alpha}$. The two critical preference parameters are agents' discount factor, $\beta$, and the weight on leisure in the utility function, $A$. The technology parameters are the depreciation rate, $\delta$, and the elasticity of output with respect to capital, $\alpha$. The technology shock, $z_{t}$, is assumed to follow an autoregressive process with autoregressive parameter $\rho$ and innovations such that $E\left(z_{t}\right)=1$.

This maximization problem produces two necessary conditions representing the laborleisure trade-off and the consumption-investment decision:

$$
c_{t}=\frac{(1-\alpha)}{A} z_{t} k_{t}^{\alpha} h_{t}^{-\alpha}
$$




$$
c_{t}^{-1}=\beta E_{t}\left[c_{t+1}^{-1}\left(\alpha z_{t+1} k_{t+1}^{\alpha-1} h_{t+1}^{1-\alpha}+1-\delta\right)\right]
$$

The solution to this economy is represented by functions for capital (i.e. investment), consumption, and labor that satisfy the two necessary conditions (eqs.(3),(4)), the economywide resource constraint, eq. (2) and are consistent with expectations implied by the Markov process for the technology shock; the arguments of these functions are the state variables $k_{t}$ and $z_{t}$. A typical method to find this solution is to "linearize" these three equations by taking a first-order Taylor series expansion of the expressions around the steady-state values. The resulting set of linear expectational difference equations implies that the solution functions will also be linear.

The first step in solving the model involves choosing values for the parameters describing tastes and technology - that is, the model is calibrated. As is well know, this is typically done so that the steady-state of the model replicates some of the stylized facts of growth. For the point that is being made in this paper, it is important to note that this is usually done in a sequential manner. That is, labor's share, equal to $(1-\alpha)$, is used to pin down $\alpha$. Then the discount factor is chosen so that the implied steady state interest rate, equal to $\beta^{-1}-1$, is consistent with the data. Next, note that in steady-state (in which $z_{t}=1$, $k_{t}=\bar{k}$, and $y_{t}=\bar{y} \forall t$ ), eq. (4) implies

$$
\frac{\beta^{-1}-1+\delta}{\alpha}=\bar{k}^{\alpha-1} \bar{h}^{1-\alpha}=\frac{\bar{y}}{\bar{k}}
$$

Therefore, the observed capital-output ratio in the economy is used to determine $\delta$. 
The final parameter, $A$, is determined by exploiting the steady-state relationship implied by eq. (3):

$$
\bar{h}=\frac{(1-\alpha)}{A} \frac{\bar{y}}{\bar{c}}
$$

Since all terms on the right-hand side other than $A$ have been determined, the choice of this parameter pins down the amount of time spent in work activity in the steady-state.

Authors that use the linearization approximation to solve real business cycle models have calibrated their models along these lines and, in particular, have stressed the importance of calibrating the weight of leisure in the utility function, i.e. the parameter $A$. Some typical examples are:

The model has to be calibrated. The necessary information can sometimes by obtained from data on individuals or households. An example of such information is the average fraction of discretionary time household members who are, or who potentially are, labor market participants actual spent in market activity. (Kydland and Prescott, 1991).

The parameter A is chosen so that the steady-state level of hours worked is exactly $h=1 / 3$, which matches the fraction of discretionary time spent in market work found in time-use studies...(Hansen and Wright, 1992).

Given an estimate of $h$, the fraction of time devoted to market activities, equation (32) (the equivalent of eq. (6)) provides the basis for calibrating the 
preference parameter, $\alpha$, based on the steady-state output consumption ratio. The value of $h$ is determined by microeconomic evidence from time allocation studies. (Cooley and Prescott, 1995).

Given these representative statements, one would surmise that this parameter is important in determining equilibrium characteristics of the model. I now demonstrate that such a conclusion is incorrect - the parameter $A$ has absolutely no influence on equilibrium behavior.

\section{Equilibrium}

This can be seen by rewriting the equations defining equilibrium as follows:

$$
\begin{gathered}
h_{t}=\frac{(1-\alpha)}{A} \frac{y_{t}}{c_{t}} \\
c_{t}^{-1}=\beta E_{t}\left[c_{t+1}^{-1}\left(\alpha \frac{y_{t+1}}{k_{t+1}}+1-\delta\right)\right] \\
k_{t+1}=y_{t}+k_{t}(1-\delta)-c_{t} \\
\rho z_{t}=E_{t}\left(z_{t+1}\right)
\end{gathered}
$$

Changes in the parameter $A$ will influence equilibrium behavior through two possible channels - directly through its presence in an equation (as in eq. (7)) or indirectly through its impact on the steady-state values around which the equations are being linearized. But linearizing the equations and expressing the variables as percentage deviations from steadystate values is akin to taking $\log$ approximations so the direct effect of parameter $A$ in eq. (7) 
will drop out. As seen in eqs. (7) and (8), the effect on steady-state values is mitigated since the capital-output ratio and output-consumption ratio are both independent of $A$. However, the steady-state values of $\left(y_{t}, c_{t}, k_{t}\right)$ are all affected by $A$ so that the second channel is still possible in the remaining equations.

To see that this channel is not present in the linearized model, replace $h_{t}$ in eq. (7) using the production function so that this equilibrium condition is now written in terms of $y_{t}$ :

$$
y_{t}=\left(\frac{1-\alpha}{A}\right)^{1 / \alpha} z_{t}^{1 / \alpha} k_{t} c_{t}^{-\left(\frac{1-\alpha}{\alpha}\right)}
$$

Then linearizing the system of equations (11), (8), (9), and (10) around the steadystate values yields the following system of equations: (Note all variables are expressed as percentage deviations from steady-state, $\left.\hat{x}_{t} \equiv\left(x_{t}-\bar{x}\right) / \bar{x}\right)$.

$$
\begin{gathered}
\hat{y}_{t}=-\frac{1-\alpha}{\alpha} \hat{c}_{t}+\hat{k}_{t}+\frac{1}{\alpha} \hat{z}_{t} \\
\hat{c}_{t}=E_{t}\left[\hat{c}_{t+1}\right]+\alpha \beta \overline{\bar{y}} \hat{k}_{t+1}-\alpha \beta \frac{\bar{y}}{\bar{k}} E_{t}\left[\hat{y}_{t+1}\right] \\
-\frac{\bar{c}}{\bar{k}} \hat{c}_{t}+(1-\delta) \hat{k}_{t}+\frac{\bar{y}}{\bar{k}} \hat{y}_{t}=\hat{k}_{t+1} \\
\rho \hat{z}_{t}=E_{t}\left[\hat{z}_{t+1}\right]
\end{gathered}
$$

As is clear, all coefficients in this system of equations are independent of the parameter $A$. Consequently, the implied solutions, i.e. the functions describing equilibrium consumption, capital (or investment) and output will also be independent of $A$. Furthermore, due to eq. (7), this implies that equilibrium labor will also be independent of this parameter.

Hence, the exercise of choosing this parameter so that the steady-state features of the model match the data equivalent is a meaningless step with regard to the equilibrium char- 
acteristics of the model. ${ }^{1} \quad$ Rather than the parameterization of the utility function, it is the functional form that drives equilibrium behavior. It is curious why this irrelevance has not been noted in previous work; however, it is possible that this is due to the numerical approximation method employed by some RBC analysts. Namely, the approach used in the early literature was to take a second-order approximation of the utility function around the steady-state. The resulting quadratic objective function implies linear necessary conditions; the linear conditions analyzed here, however, would imply, up to a constant, the same objective function. But working with the quadratic objective function may make these relationships, and, in particular, the irrelevance of the parameter $A$, less transparent. ${ }^{2}$

As stated earlier, the use numerical methods to solve economic models is highly beneficial since it greatly expands the environments which can be studied. However, it is important to analytically investigate the structure of these models so that our intuition is enhanced.

\footnotetext{
${ }^{1}$ It is important to point out that this result does not generalize to all preferences; in particular, if the utility of leisure is not linear, then the weighting parameter will influence cyclical behavior. The critical feature in this model is that eq.(7) is linear in labor.

2 Another possible reason for why this result was not noted in the early literature is that the policy functions were often defined in terms of the levels of the variables rather than percentage deviations from steady-state. Consequently, the coefficients on the equilibrium policy function, in particular, the constant term, would change as the value of $A$ changed. But since the end analysis typically involves percentage deviations from trend (defined by the H-P filter), this difference is eliminated.
} 


\section{References}

Cooley, T. F. and E. C. Prescott (1995) "Economic growth and business cycles," in: T. F. Cooley (Ed.) Frontiers of Business Cycle Research (Princeton, Princeton University Press).

Hansen, G. D. (1985) "Indivisible labor and the business cycle," Journal of Monetary Economics 16, 309-27.

Hansen, G. D. and R. Wright (1992), "The labor market in real business cycle theory," Federal Reserve Bank of Minneapolis Quarterly Review, 2-12.

Kydland, F. E. and E. C. Prescott (1991) "The econometrics of the general equilibrium approach to business cycles," Scandinavian Journal of Economics 93, 161-178. 